\title{
Tanshinone IIA inhibits osteoclast differentiation through down-regulation of c-Fos and NFATc1
}

\author{
Han Bok Kwak ${ }^{1}$, Daum Yang ${ }^{1}$, \\ Hyunil $\mathrm{Ha}^{1}$, Jong-Ho Lee ${ }^{1}$, \\ Ha-Neui Kim ${ }^{1}$, Eun-Ran Woo', \\ Seungbok Lee ${ }^{1}$, Hong-Hee $\mathrm{Kim}^{1}$ \\ and Zang Hee Lee ${ }^{1,3}$ \\ ${ }^{1}$ Department of Cell and Developmental Biology \\ School of Dentistry, DRI, and BK21 Program \\ Seoul National University \\ Seoul 110-749, Korea \\ ${ }^{2}$ Research Center for Proteineous Materials \\ Chosun University \\ Gwangju 501-759, Korea \\ ${ }^{3}$ Corresponding author: Tel, 82-2-740-8672; \\ Fax, 82-2-747-6589; E-mail, zang1959@hotmail.com
}

Accepted 23 May 2006

Abbreviations: M-CSF, macrophage CSF; OVX, ovariectomy; RANKL, receptor activator of NF- $\mathrm{KB}$ ligand; RT-PCR, reverse transcription-PCR

\begin{abstract}
Bone is a dynamic tissue that is regulated by the activity of bone-resorbing osteoclasts and boneforming osteoblasts. Excessive osteoclast formation causes diseases such as osteoporosis and rheumatoid arthritis. Natural substances may be useful as therapeutic drugs to prevent many diseases in humans because they avoid the many side effects of treatment with chemical compounds. Here we show that tanshinone IIA isolated from Salvia miltiorrhiza Bunge inhibits the receptor activator of NF- $\kappa B$ ligand (RANKL)-mediated osteoclast differen tiation of osteoclast precursors. Tanshinone IIA suppressed the expression levels of c-Fos and NFATc1 induced by RANKL. However, retrovirusmediated overexpression of c-Fos induced the expression of NFATc1 despite the presence of tans hinone IIA and reversed the inhibitory effect of tanshinone IIA on osteoclast differentiation. Also, the introduction of osteoclast precursors with the NFATc1 retrovirus led to osteoclast differentiation in the presence of tanshinone IIA. Our results suggest that tanshinone IIA may have a role as a therapeutic drug in the treatment of bone disease such as
\end{abstract}

osteoporosis.

Keywords: osteoclasts; tanshinone; Salvia miltiorrhiza; drugs, Chinese herbal; osteoporosis; RANK ligand

\section{Introduction}

The balance between bone-resorbing osteoclasts and bone-forming osteoblasts is important to bone homeostasis. Many factors tightly regulate bone resorption and bone formation in vivo to maintain bone homeostasis (Suda et al., 1999; Boyle et al., 2003). Bone erosion through excessive osteoclast formation is associated with alterations including estrogen deficiency and increases in inflammatory cytokines (Abu-Amer et al., 1997; Miyaura et al., 2003) and in turn causes bone diseases such as rheumatoid arthritis, periodontal disease, osteoporosis, and periprosthetic osteolysis. In addition, under certain conditions such as aging or prolonged smoking, bone loss is caused by a defect in the boneforming osteoblasts (Merkel et al., 1999; Liu et al., 2001; Redlich et al., 2002). Most therapeutic drugs currently used to treat bone disease inhibit osteoclast formation or bone resorption. Consequently, there is a need for effective therapeutic drugs that improve the quality and quantity of bone.

Osteoclasts, which are the cells primarily responsible for bone resorption, are of hamatopoietic origin. Osteoclast differentiation is regulated by multiple processes through differential gene expression, one of which is characterized by the fusion of osteoclast precursors. Osteotropic agents including, IL-1, IL-6, IL-11, IL-15, IL-17, TNF- $\alpha$, PGE2 and PTH cause bone loss by increasing osteoclast formation (Suda et al., 1999; Hofbauer et al., 2000). Receptor activator of NF-KB ligand (RANKL), which is an essential osteoclastogenic cytokine, recruits TNF receptor-associated factor (TRAF) proteins through binding to its receptor, RANK, on the surface of osteoclast precursors. TRAFs activate various signaling pathways, including C-Src, PI3-kinase/Akt, and MAPK (Anderson et al., 1997; Lee and Kim, 2003). Several transcription factors, including NF-kB, C-Fos, NFATC1, PU1 and MITF, are activated by RANKL and are important for osteoclast differentiation (Teitelbaum et al., 2003). In particular, expression of c-Fos induced by RANKL plays an 
essential role in the initiation of osteoclast differentiation. c-Fos causes the expression of NFATc1 during RANKL-mediated osteoclast differentiation (Matsuo et al., 2004). NFATc1 can induce osteoclast differentiation in the absence of RANKL. Thus, NFATc1 is an essential factor in osteoclast differentiation (Takayanagi et al., 2002).

Tanshinones are the major component of Danshen, the dried roots of Salvia miltiorrhiza Bunge (Labiatae) that is used as a drug in traditional Chinese medicine (Hazra et al., 2004; Hur et al., 2005). Tanshinones have been widely used to treat menorrhalgia, insomnia, coronary disease, and cardiovascular disease (Takahashi et al., 2002; Han et al., 2005). Furthermore, tanshinone IIA has been shown to be a potent antioxidant and to have anticancer activity against various cancer cells (Wu et al., 1998; Wang et al., 2005). Although tanshinone IIA has been used as a medicinal agent in the treatment of many diseases, its role in osteoclast-related bone disease remains unknown.

We showed previously that tanshinone IIA greatly inhibits osteoclast differentiation and suppresses bone resorption through disruption of the actin ring (Kim et al., 2004). Here we examine the precise inhibitory mechanism of tanshinone IIA on RANKLinduced osteoclast differentiation. This study shows that tanshinone IIA prevents osteoclast differentiation by inhibiting c-Fos and NFATc1 expression.

\section{Materials and Methods}

\section{Reagents}

Tanshinone IIA was isolated from Danshen as described previously (Kim et al., 2004). Recombinant human RANKL and macrophage CSF (M-CSF) were purchased from PeproTech EC (London, England). Antibody to c-Fos was obtained from Cell Signaling Technology (Beverly, MA). Antibodies to NFATc1 and actin were purchased from Santa Cruz Biotechnology (Santa Cruz, CA). pMX-IRES-EGFP, pMXc-Fos-IRES-EGFP, and pMX-NFATc1-IRES-EGFP vectors were kindly provided by Dr. Nacksung Kim (University of Chonnam).

\section{Isolation of osteoclast precursors and osteoclastogenesis}

Osteoclast precursors were prepared as previously described with some modification (Park et al., 2005). Briefly, bone marrow cells were obtained from the tibia and femur of 5 -week-old male ICR mice. Bone marrow cells were suspended in $\alpha$-MEM (Welgene) containing 10\% FBS (Gibco BRL) and antibiotics (Sigma) and were cultured for 1 day in the presence of M-CSF (10 ng/ml). After 1 day, nonadherent cells were seeded on a $10-\mathrm{cm}$ bacterial culture dishes and cultured for 3 days in the presence of M-CSF $(30 \mathrm{ng} / \mathrm{ml})$ and further adherent cells were used as osteoclast precursors. For the osteoclast differentiation assay, osteoclast precursors were cultured for 4 days with M-CSF (30 ng/ml) and RANKL (50 ng/ml) in the presence or absence of tanshinone IIA $(10 \mu \mathrm{g} /$ $\mathrm{ml}$ ). Alternatively, calvarial osteoblasts were isolated from 1-day-old ICR mouse as described previously (Kwak et al., 2004). Briefly, mouse calvariae were obtained from pericranium and washed with HBSS containing antibiotics. Calvariae were digested 5 times for $15 \mathrm{~min}$ in $0.1 \%$ collagenase and $0.2 \%$ dispase. Cells were cultured for 3 days and adherent cells were used as osteoblasts. Osteoblasts and bone marrow cells were cocultured for 6 days with TNF- $\alpha(20 \mathrm{ng} / \mathrm{ml}), \mathrm{IL}-1 \alpha(10 \mathrm{ng} / \mathrm{ml})$, or LPS $(1 \mu \mathrm{g} / \mathrm{ml})$ in the presence or absence of tanshinone IIA (10 $\mu \mathrm{g} / \mathrm{ml})$.

\section{Reverse transcription (RT)-PCR analysis}

For RT-PCR assays, total RNA was isolated by using the TRI reagent (Invitrogen, Carlsbad, CA) according to the manufacturer's instructions. Two micrograms of total RNA from each sample was reverse transcribed in a mixture containing oligo dT primer, dNTP, reverse transcriptase buffer, RNase out, DTT, and Superscript II reverse transcriptase (Invitrogen). cDNA (1 $\mu \mathrm{l})$ was amplified by PCR to generate the genes listed as follows: RANK sense, 5'-CACAGACAAATGCAAACCTTG-3; RANK antisense, 5'-GTGTTCTGGAACCTATCTTCCTCC-3'; c-Fms sense, 5'-AGCTCTCAGTACTTCAGGGC-3'; c-Fms antisense, 5'-CAAAGGCACCGGCTCCTAGA-3'; c-Fos sense, 5'-CTGGTGCAGCCCACTCTGGTC-3'; c-Fos antisense, 5'-CTTTCAGCAGATTGGCAATCTC-3'; NFATc1 sense, 5'-CAACGCCCTGACCACCGATAG-3'; NFATc1 antisense, 5'-GGCTGCCTTCCGTCTCATAGT-3'. PCR amplification was performed for $20-25$ cycles at $94^{\circ} \mathrm{C}$ for $30 \mathrm{~s}, 56-58^{\circ} \mathrm{C}$ for $30 \mathrm{~s}$, and $72^{\circ} \mathrm{C}$ for $30 \mathrm{~s}$, followed by a $7 \mathrm{~min}$ at $72^{\circ} \mathrm{C}$. PCR product were separated on a $1 \%$ agarose gels and visualized by staining with ethidium bromide. Densitometric values for each band were quantified by using the Image Pro-plus program version 4.0 (Media Cybernetics).

\section{Immunoblotting}

Cells were lysed in lysis buffer $(50 \mathrm{mM}$ Tris-Cl, 150 $\mathrm{mM} \mathrm{NaCl}, 5 \mathrm{mM}$ EDTA, 1\% Triton X-100, $1 \mathrm{mM} \mathrm{NaF}$, $1 \mathrm{mM} \mathrm{Na}_{3} \mathrm{VO}_{4}$, and $1 \%$ deoxycholate) containing protease inhibitors. Cell lysates were centrifuged at $16,000 \times g$ for $20 \mathrm{~min}$, and total protein was measured with a DC Protein Assay kit (Bio-Rad, Hercules, CA). Equal amounts of protein were electro- 
phoresed by $10-15 \%$ SDS-PAGE and transferred to a polyvinylidene difluoride membrane (Amersham Biosciences, Piscataway, NJ). Protein levels were analyzed by immunoblotting with antibodies to c-Fos, NFATc1, and actin that were detected by using enhanced chemiluminescent reagents.

\section{Retrovirus preparation}

For retrovirus preparation, Plat E packaging cells were transiently transfected with pMX-IRES-EGFP, pMX-c-Fos-IRES-EGFP, or pMX-NFATc1-IRES-EGFP vectors by using Lipofectamine 2000 as recommended by the manufacturer. Six to eight hour after transfection, the medium was replaced with DMEM containing $10 \%$ FBS and antibiotics. Culture super- natant fluid containing retrovirus was collected $48 \mathrm{~h}$ after transfection and was used as a retrovirus.

\section{Luciferase assay}

NFATc1 luciferase reporter gene was kindly provided by Dr. Nacksung Kim (University of Chonnam). HEK 293T cells were plated in 24-well plates and transiently transfected with RANK and the AP-1 or NFATc1 leuciferase reporter genes using Lipofectamine 2000 and incubated for $24 \mathrm{~h}$ with or without tanshinone IIA. The cells were lysed with lysis buffer, and the luciferase activities were measured using a luciferase reporter assay system (Promega, Madison, WI).

A
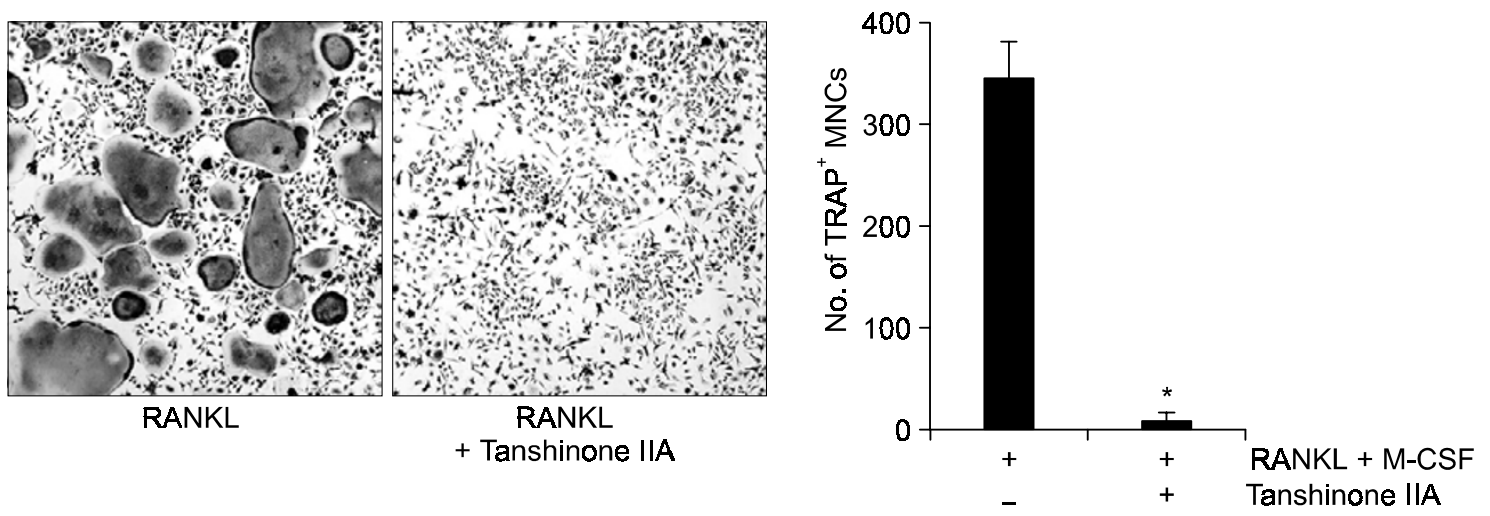

B

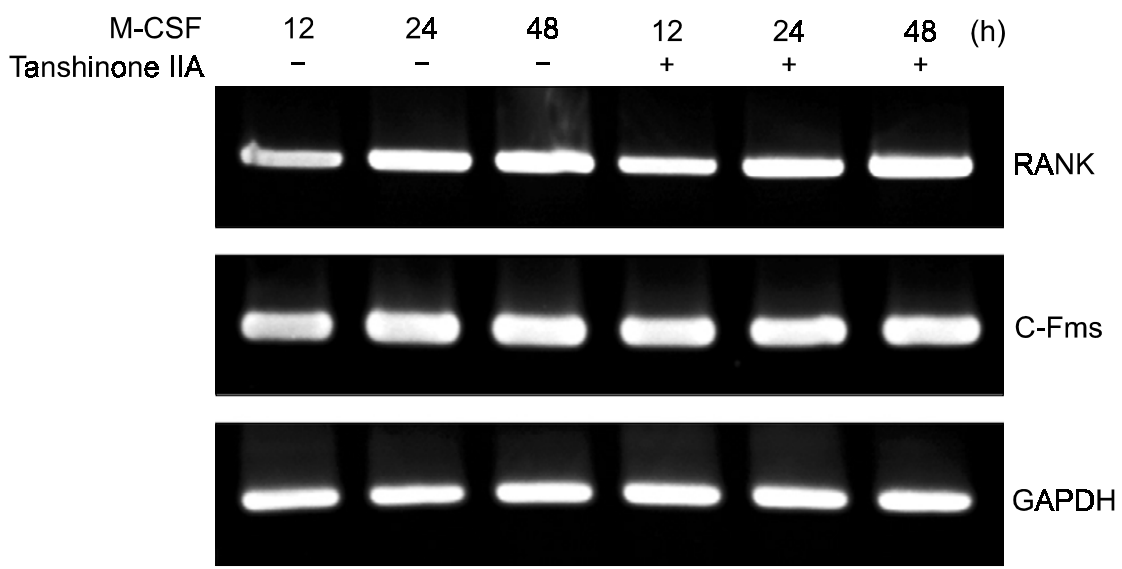

Figure 1. Inhibition of RANKL-mediated osteoclastogenesis by tanshinone IIA. (A) Osteoclast precursors were cultured with M-CSF (30 $\mathrm{ng} / \mathrm{ml})$ and RANKL $(50 \mathrm{ng} / \mathrm{ml})$ for 4 days in the presence or absence of tanshinone IIA. Cells were fixed in $10 \%$ formalin, permeabilized with $0.1 \%$ Triton X-100, and stained with TRAP solution. (B) TRAP ${ }^{+}$cells containing five or more nuclei were counted as multinucleated osteoclasts. Data from three experiments are presented as mean \pm S.D. ${ }^{*} P<0.01$, significantly different from the control. (C) Osteoclast precursors were incubated with M-CSF $(30 \mathrm{ng} / \mathrm{ml})$ in the presence or absence of tanshinone IIA for the indicated time. The mRNA expression of the indicated genes was analyzed by RT-PCR. 


\section{Statistical analysis}

All quantitative results are presented as means \pm S.D. Statistical significance was analyzed by using Student's $t$ test. $P$ values $<0.05$ were considered to be statistically significant and are defined with an asterisk.

\section{Results}

\section{Tanshinone IIA inhibits RANKL-mediated osteoclast differentiation}

Tanshinone IIA greatly suppressed RANKL-mediated osteoclast differentiation in osteoclast precursors (Figure $1 \mathrm{~A}$ and $\mathrm{B}$ ). We also examined the alteration of expression of RANK and c-Fms, which act as receptors for RANKL and M-CSF, respectively. Osteoclast precursors were treated with DMSO or tanshinone IIA for various times in the presence of M-CSF. M-CSF increased RANK mRNA expression in osteoclast precursors, as previously reported (Mi- yamoto et al., 2000). Tanshinone IIA did not change the expression of RANK and C-Fms in osteoclast precursors (Figure $1 \mathrm{C}$ ). These results suggest that tanshinone IIA does not inhibit signaling pathways, which involved in M-CSF-induced RANK expression and that tanshinone IIA may affect RANKL-induced signaling involved in osteoclastogenesis.

\section{Tanshinone IIA suppresses c-Fos and NFATc1 expression induced by RANKL}

Osteoclast differentiation is regulated through the induction of various genes in response to RANKL. In particular, c-Fos and NFATc1 are well known to play an essential role in the differentiation of osteoclast precursors (Takayanagi et al., 2002; Hirotani et al., 2004). We thus studied whether tanshinone IIA regulates the expression of c-Fos and NFATc1 in response to RANKL. Osteoclast precursors were pretreated with DMSO or tanshinone IIA $(10 \mu \mathrm{g} / \mathrm{ml})$ and further stimulated with RANKL $(100 \mathrm{ng} / \mathrm{ml})$ for various times in the presence of M-CSF. Densito-
A

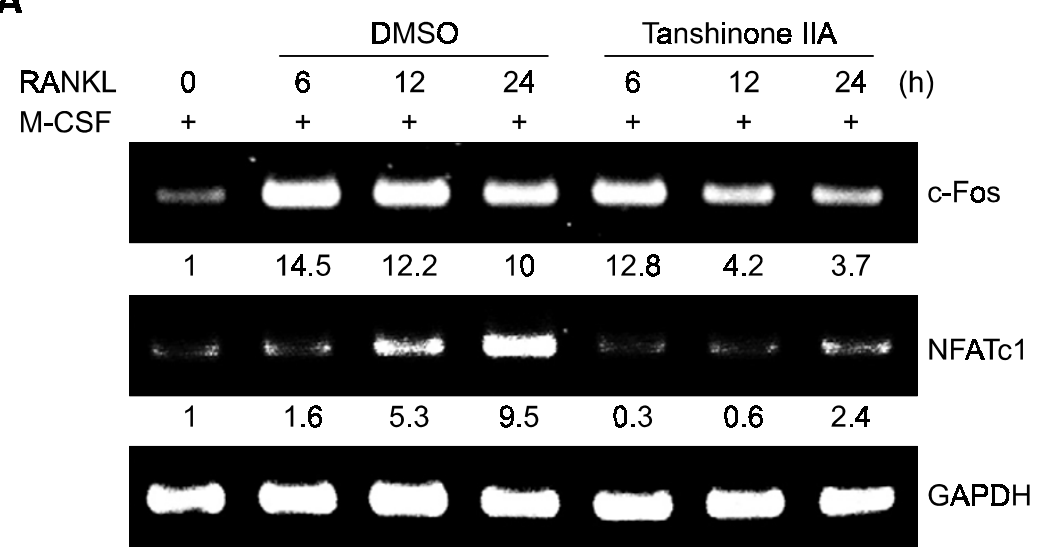

B

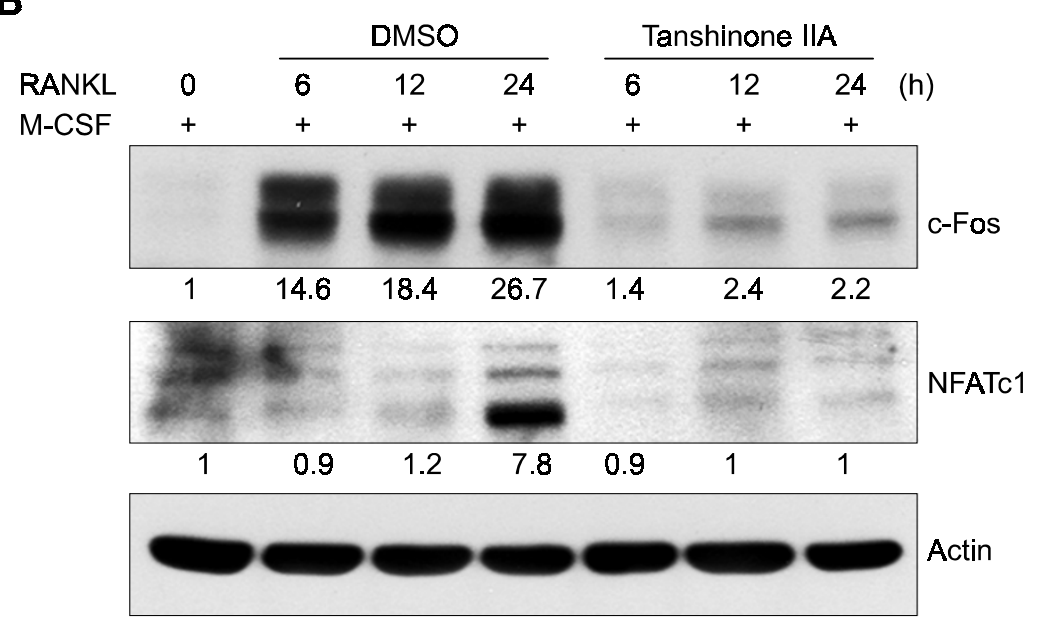

Figure 2. Inhibition of RANKL-induced c-Fos and NFAT2 expression in osteoclast precursors by tanshinone IIA. (A) Osteoclast precursors were pretreated for $1 \mathrm{~h}$ with or without tanshinone IIA and then stimulated with RANKL $(100 \mathrm{ng} / \mathrm{ml})$ in the presence of M-CSF $(30 \mathrm{ng} / \mathrm{ml})$ for the indicated time. Total RNA was isolated and reverse transcribed, and the mRNA expression of the indicated genes was measured by RT-PCR. (B) Cells were stimulated as in (A). Cells lysates were immunoblotted with antibodies to p38 and NFATc1 to detect c-Fos and NFATc1. Band intensity was quantified by using an Image Pro-plus program version 4.0 (Media Cybernetics). 
A

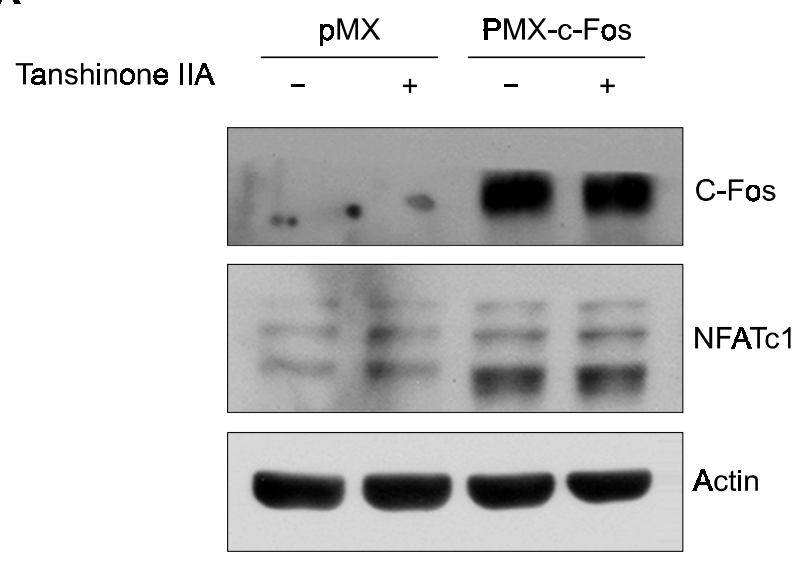

C

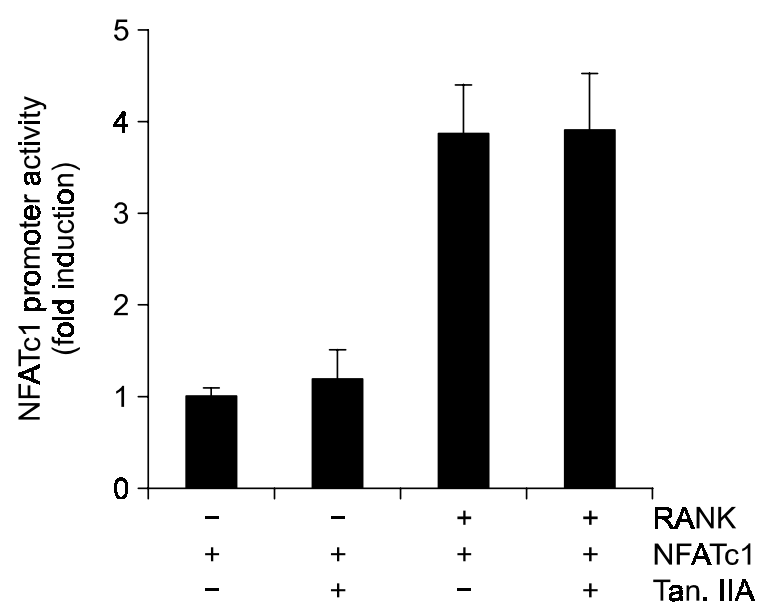

B

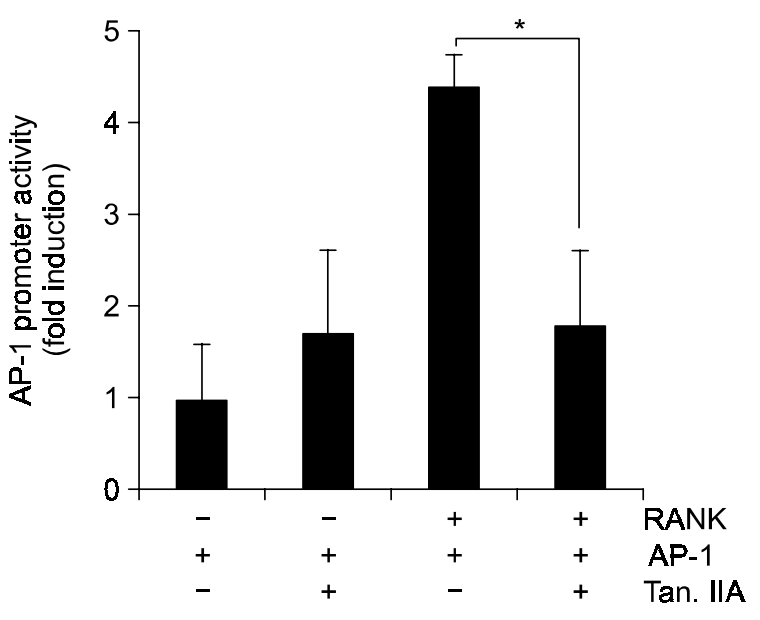

E

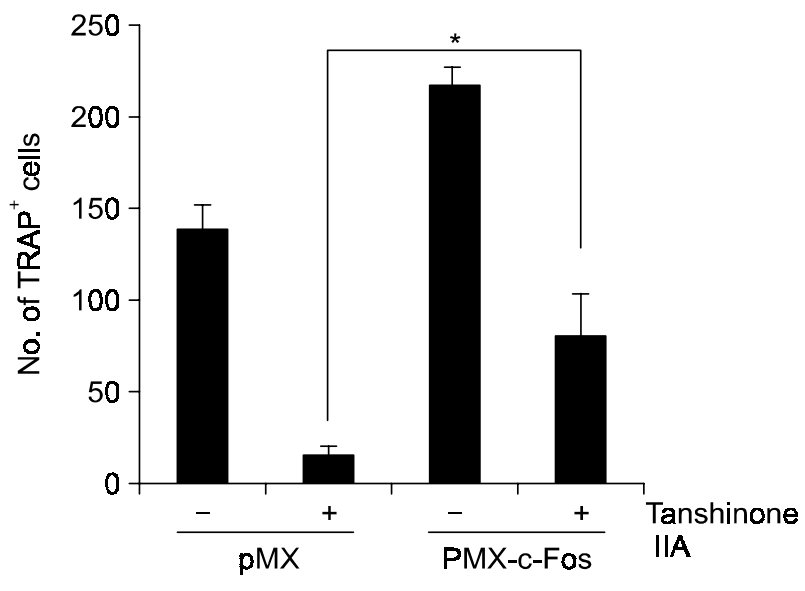

D

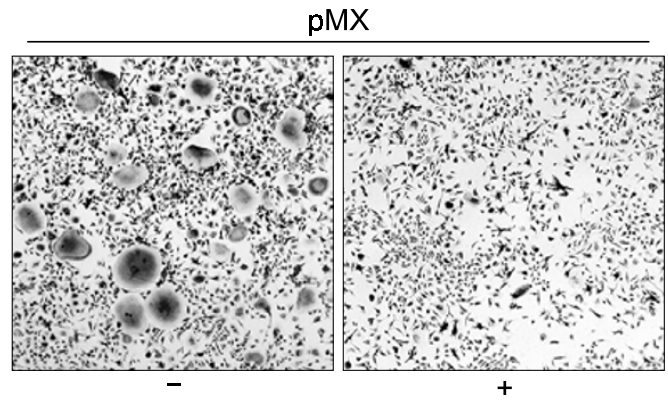

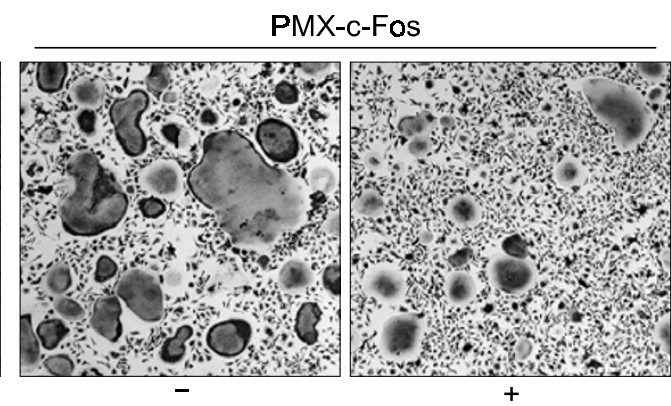

Tan. IIA

Figure 3. Overexpression of c-Fos reverses the inhibitory effect of tanshinone IIA on osteoclast differentiation. pMX-IRES-EGFP or pMXc-Fos-IRES-EGFP vectors were transiently transfected into Plat $\mathrm{E}$ cells. After 6-8 $\mathrm{h}$, the medium was changed and the culture supernatant fluid was used as a retrovirus as described in Materials and methods. (A) Osteoclast precursors were infected with control or c-Fos retrovirus for $7 \mathrm{~h}$ in the presence of M-CSF $(30 \mathrm{ng} / \mathrm{ml})$ and polybrene $(6 \mu \mathrm{g} / \mathrm{ml})$. The medium was replaced and further treated with or without tanshinone IIA (10 $\mu \mathrm{g} / \mathrm{ml})$ for $48 \mathrm{~h}$. Cell lysates were immunoblotted with antibodies to c-Fos and NFATc1. (B) HEK 293T cells were seeded in a 24-well plates and transiently transfected with AP-1 reporter gene or RANK and AP-1 reporter gene. Cells were incubated for $24 \mathrm{~h}$ in the presence or absence of tanshinone IIA. 24 $\mathrm{h}$ after transfection, the cells were lysed and assayed for luciferase activity using a luciferase reporter assay system. (C) HEK 293T cells were transiently transfected with NFATc1 reporter gene or RANK and NFATc1 reporter gene. Luciferase assay was performed as above. (D) Osteoclast precursors were infected with control or c-Fos retrovirus as in (A) and were then cultured for 5 days with M-CSF (30 ng/ml) and RANKL (100 ng/ml) in the presence or absence of tanshinone IIA $(10 \mu \mathrm{g} / \mathrm{ml})$. Cells were fixed and stained for TRAP. (E) TRAP ${ }^{+}$cells were counted as osteoclasts. 
metric value of RT-PCR normalized by GAPDH intensity showed that tanshinone IIA greatly inhibited the expression of NFATc1 mRNA and slightly suppressed c-Fos mRNA expression in response to RANKL (Figure 2A). Consistent with the results of the RT-PCR analyses, protein levels of c-Fos and NFATc1 were increased in response to RANKL, but the expression of both c-Fos and NFATc1 was significantly inhibited by tanshinone IIA (Figure 2B). Thus, tanshinone IIA may inhibit osteoclast differentiation by inhibiting c-Fos and NFATc1 expression in response to RANKL.
Inhibition of osteoclast differentiation by tanshinone IIA is reversed by overexpression of c-Fos

To elucidate the mechanism by which tanshinone IIA inhibits c-Fos and NFATc1 expression, we infected osteoclast precursors with control retrovirus or c-Fos retrovirus for $7 \mathrm{~h}$ in the presence of M-CSF (30 $\mathrm{ng} / \mathrm{ml})$ and polybrene $(6 \mu \mathrm{g} / \mathrm{ml})$. The medium was replaced with fresh $\alpha$-MEM containing $10 \%$ FBS and antibiotics and further cultured for $48 \mathrm{~h}$ with M-CSF in the presence or absence of tanshinone IIA. As shown in Figure $3 \mathrm{~A}$, the expression of $\mathrm{c}-\mathrm{Fos}$ and NFATc1 induced by c-Fos retroviral infection com-

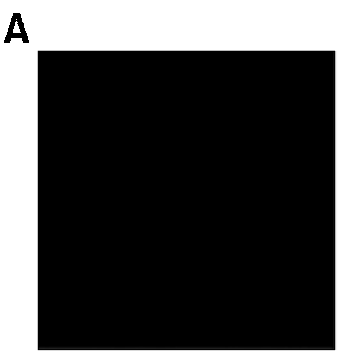

Control

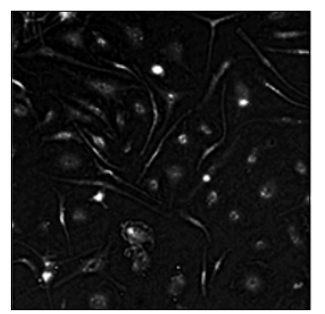

$\mathrm{pMX}$

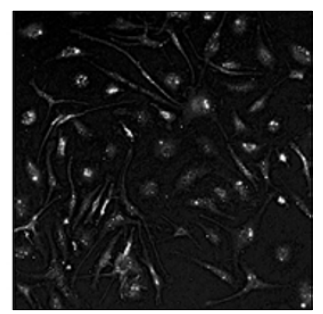

NFATc1
B

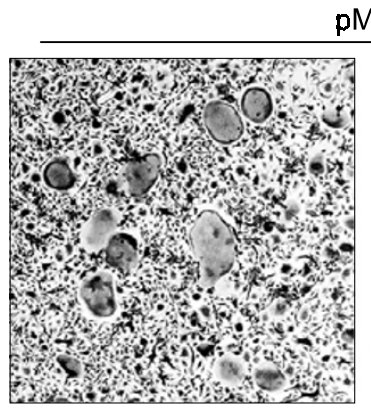

$-$
$\mathrm{pMX}$

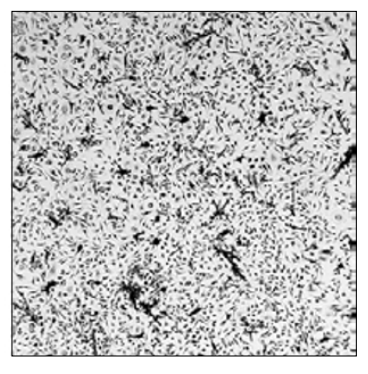

$+$
C

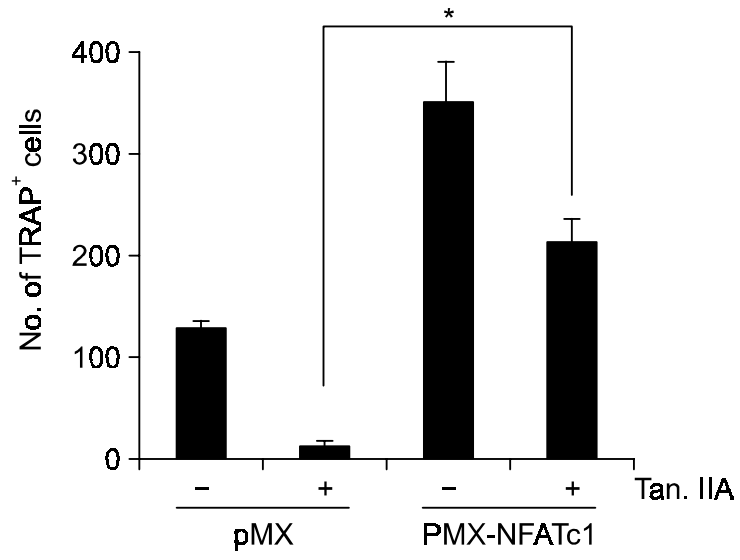

PMX-NFATc1

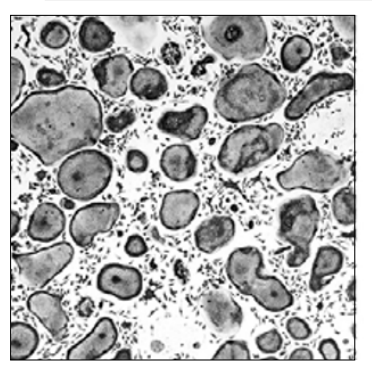

-

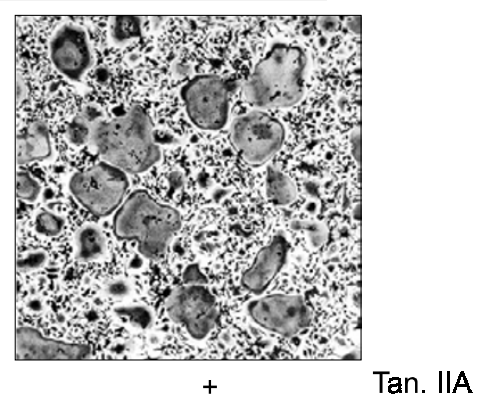

Figure 4. Overexpression of NFATc1 reverses the effect of tanshinone IIA in osteoclast differentiation. Plat $\mathrm{E}$ cells were transiently transfected with pMX-NFATc1-IRES-EGFP or pMX-IRES-EGFP vectors to obtain retrovirus. (A) Osteoclast precursors were infected with control retrovirus or NFATc1 retrovirus for $7 \mathrm{~h}$ in the presence of M-CSF $(30 \mathrm{ng} / \mathrm{ml})$ and polybrene $(6$ $\mu \mathrm{g} / \mathrm{ml})$. Infected cells were visualized by confocal microscopy. (B) Osteoclast precursors were infected with control or NFATc1 retrovirus as in (A) and then cultured for 5 days with M-CSF $(30 \mathrm{ng} / \mathrm{ml})$ and RANKL $(100 \mathrm{ng} / \mathrm{ml})$ in the presence or absence of tanshinone IIA $(10 \mu \mathrm{g} / \mathrm{ml})$. Cells were fixed and stained for TRAP. (C) TRAP ${ }^{+}$cells were counted as osteoclasts. 
pletely rescued the inhibitory effect of tanshinone IIA. Furthermore, we examined for direct evidence that tanshinone IIA inhibits the c-Fos and NFATc1 transcriptional activity by cotransfecting HEK 293T cells with RANK and the AP-1 or NFATc1 promoterluciferase gene. RANK-mediated AP-1 promoter activity was inhibited in the presence of tanshinone IIA. However, tanshinone IIA does not inhibit NFATc1 transcriptional activity (Figure $3 \mathrm{~B}$ and $\mathrm{C}$ ). These results suggest that tanshinone IIA specifically regulates c-Fos expression in response to RANKL rather than regulating NFATc1 expression.

Next we investigated whether overexpression of c-Fos in osteoclast precursors could rescue the inhibition of osteoclast differentiation by tanshinone IIA. After infection with control or c-Fos retrovirus, cells were cultured for 5 days with M-CSF and RANKL in the presence or absence of tanshinone IIA. Osteoclast differentiation was significantly higher after infection of osteoclast precursors with c-Fos retrovirus than after infection with control retrovirus in the presence of tanshinone IIA (Figure 3D and E). These results suggest that tanshinone IIA suppresses osteoclast differentiation through inhibition of c-Fos expression.

\section{NFATc1 rescues osteoclast differentiation in the presence of tanshinone IIA}

The induction of NFATc1 in osteoclast precursors is essential for osteoclast differentiation and is regulated by both c-Fos and the calcium/calcineurin pathways (Takayanagi et al., 2002). Inhibition of NFATc1 by tanshinone IIA is associated with inhibition of c-Fos in response to RANKL. Therefore, we examined whether NFATc1 could overcome the inhibition of osteoclast differentiation caused by tanshinone IIA. After infection of osteoclast precursors with control and NFATc1 retrovirus, the medium was replaced with fresh $\alpha$-MEM containing $10 \%$ FBS and antibiotics and further cultured for $48 \mathrm{~h}$ with M-CSF. GFP-expressed cells were visualized by confocal microscopy, and the infection efficiency of the control and NFATc1 retroviruses was more than 90\% (Figure 4A). Osteoclast precursors were infected with control or NFATc1 retrovirus in the presence of M-CSF $(30 \mathrm{ng} / \mathrm{ml})$ and polybrene $(6 \mu \mathrm{g} / \mathrm{ml})$ and further cultured for 5 days with M-CSF $(30 \mathrm{ng} / \mathrm{ml})$ and RANKL $(50 \mathrm{ng} / \mathrm{ml})$ in the presence or absence of tanshinone IIA $(10 \mu \mathrm{g} / \mathrm{ml})$. As shown in Figure 4B and $\mathrm{C}$, overexpression of NFATc1 rescued the blockage of osteoclast differentiation in the presence of tanshinone IIA. Thus, the inhibitory effects of tanshinone IIA on osteoclast differentiation result from the decrease in NFATc1 by inhibition of c-Fos.

\section{Discussion}

Bone is a dynamic tissue that is maintained by the balance between bone formation and bone resorption; this balance can be easily upset by chronic inflammation, menopause, and aging (Karsenty et al., 2002). Bone erosion results from excessive osteoclast formation rather than damage to osteoblast activity. Therefore, the development of drugs that act to regulate osteoclast formation is important to prevent bone disease. Previously, we reported that tanshinone IIA significantly inhibits the differentiation of osteoclast precursors mediated by RANKL (Kim et al., 2004). Two key factors, RANKL and M-CSF, regulate the expression of various genes in osteoclast precursors by binding to their receptors, RANK and c-Fms, respectively, the levels of which are important in osteoclast differentiation. In particular, expression of c-Fms is a crucial feature of osteoclast progenitors. The addition of M-CSF to osteoclast progenitors causes the expression of RANK; thereby, RANKL induces differentiation into osteoclasts (Miyamoto et al., 2000). Although tanshinone IIA inhibits osteoclast differentiation, it does not alter the expression of RANK or c-Fms (Figure 1). These results suggest that tanshinone IIA suppresses osteoclast differentiation induced by RANKL.

A member of the TNF superfamily, RANKL mediates multiple signaling pathways via RANK in osteoclast precursors and activates critical transcription factors for osteoclast differentiation and function (Suda et al., 1999; Boyle et al., 2003; Lee and Kim, 2003). One such transcription factor is NFATc1, which is expressed in osteoclast precursors through $\mathrm{Ca}^{2+}$ oscillation and $\mathrm{c}-\mathrm{Fos}$ in response to RANKL and that is required for the regulated expression of many genes, including TRAP, calcitonin receptor, and cathepsin K (Takayanagi et al., 2005). Thus, regulation of NFATc1 expression in osteoclast precursors has significant consequences for bone disorder therapeutics. We found that the inhibition of osteoclast differentiation by tanshinone IIA coincides with regulation of c-Fos and NFATc1. Tanshinone IIA slightly inhibits the mRNA expression of endogenous c-Fos induced by RANKL but completely inhibits the expression of the c-Fos protein (Figure 2). Therefore, we hypothesized that down-regulation of c-Fos protein is involved in c-Fos mRNA degradation. However, tanshinone IIA did not affect the expression of exogenous c-Fos induced by c-Fos retroviral infection (Figure 3 ) or the stability of c-Fos mRNA (data not shown). These results indicate that tanshinone IIA does not affect the mRNA or protein stability of c-Fos or its posttranscriptional regulation. Our previous results indicate that tanshinone IIA 
causes alteration of RANKL-induced signaling pathways including ERK (Kim et al., 2004), a known signaling pathway to regulate c-Fos expression (Whitmarsh et al., 1995). Thus, this altered signaling pathway is related to inhibitory effect of tanshinone IIA on RANKL-induced c-Fos expression.

c-Fos, however, plays an important role in the expression of NFATc1, which is an essential factor for osteoclast differentiation. We also found that overexpression of c-Fos caused by c-Fos retroviral infection can rescue NFATc1 expression in the presence of tanshinone IIA (Figure 3). However, the inhibitory effect of tanshinone IIA on RANKL-mediated osteoclast differentiation was not entirely rescued by c-Fos or NFATc1 administration. Actually, NFATc1 is essential for osteoclast differentiation but is not sufficient for osteoclast differentiation in the absence of RANKL. Other transcription factors, in-

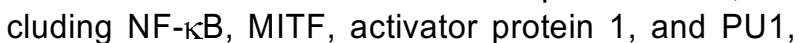
are required for osteoclast differentiation (Teitelbaum et al., 2003; Walsh et al., 2003). These results indicate that osteoclast differentiation induced by RANKL requires several transcription factors for cooperating with NFATc1. We showed previously that tanshinone IIA suppresses Akt, ERK, and NF-kB activation in response to RANKL (Kim et al., 2004). Thus, we conclude that tanshinone IIA prevents functional cooperation between NFATc1 and NF-KB in response to RANKL.

In summary, we investigated the effect of tanshinone IIA on osteoclast formation. Tanshinone IIA greatly inhibits osteoclast formation by inhibiting the expression of c-Fos and NFATc1 induced by RANKL. Taken together, our results suggest that tanshinone IIA probably plays an important role in inhibiting bone loss by preventing osteoclast formation.

\section{Acknowledgement}

This work was supported by grant R01-2005-00010073-0 from the Basic Research Program of the Korea Science and Engineering Foundation and by a grant from BioGreen 21 Program, Rural Development Administration, Republic of Korea.

\section{References}

Abu-Amer Y, Ross FP, Edwards J, Teitelbaum SL. Lipopolysaccharide-stimulated osteoclastogenesis is mediated by tumor necrosis factor via its p55 receptor. J Clin Invest 1997;100:1557-65

Anderson DM, Maraskovsky E, Billingsley WL, Dougall WC, Tometsko ME, Roux ER, Teepe MC, DuBose RF, Cosman D, Galibert L. A homologue of the TNF receptor and its ligand enhance T-cell growth and dendritic-cell function. Nature 1997;390:175-9
Boyle WJ, Simonet WS, Lacey DL. Osteoclast differentiation and activation. Nature 2003;423:337-42

Christgau S, Tanko LB, Cloos PA, Mouritzen U, Christiansen $C$, Delaisse JM, Hoegh-Andersen P. Suppression of elevated cartilage turnover in postmenopausal women and in ovariectomized rats by estrogen and a selective estrogen-receptor modulator (SERM). Menopause 2004;5: 508-18

Desimone DP, Greene VS, Hannon KS, Turner RT, Bell NH. Prostaglandin E2 administered by subcutaneous pellets causes local inflammation and systemic bone loss: a model for inflammation-induced bone disease. J Bone Miner Res 1993;8:625-34

Han YM, Oh H, Na M, Kim BS, Oh WK, Kim BY, Jeong DG, Ryu SE, Sok DE, Ahn JS. PTP1B inhibitory effect of abietane diterpenes isolated from Salvia miltiorrhiza. Biol Pharm Bull 2005;28:1795-7

Hazra B, Sarma M Das, Sanyal U. Separation methods of quinonoid constituents of plants used in Oriental traditional medicines. J Chromatogr B Analyt Technol Biomed Life Sci 2004;812:259-75

Hirotani H, Tuohy NA, Woo JT, Stern PH, Clipstone NA. The calcineurin/nuclear factor of activated T cells signaling pathway regulates osteoclastogenesis in RAW264.7 cells. J Biol Chem 2004;279:13984-92

Hofbauer LC, Dunstan CR, Lacey DL, Boyle WJ, Riggs BL. The roles of osteoprotegerin and osteoprotegerin ligand in the paracrine regulation of bone resorption. J Bone Miner Res 2000;15:2-12

Hur JM, Shim JS, Jung HJ, Kwon HJ. Cryptotanshinone but not tanshinone IIA inhibits angiogenesis in vitro. Exp Mol Med 2005;37:133-7

Karsenty G, Wagner EF. Reaching a genetic and molecular understanding of skeletal development. Dev Cell 2002; 2:389-406

Kim HH, Kim JH, Kwak HB, Huang H, Han SH, Ha H, Lee SW, Woo ER, Lee ZH. Inhibition of osteoclast differentiation and bone resorption by tanshinone IIA isolated from Salvia miltiorrhiza Bunge. Biochem Pharmacol 2004;67:1647-56

Kwak HB, Lee SW, Li YJ, Kim YA, Han SY, Jhon GJ, Kim HH, Lee ZH. Inhibition of osteoclast differentiation and bone resorption by a novel lysophosphatidylcholine derivative, SCOH. Biochem Pharmacol 2004;67:1239-48

Lean JM, Davies JT, Fuller K, Jagger CJ, Kirstein B, Partington GA, Urry ZL, Chambers TJ. A crucial role for thiol antioxidants in estrogen-deficiency bone loss. J Clin Invest 2003;112:915-23

Lee ZH, Kim HH. Signal transduction by receptor activator of nuclear factor kappa B in osteoclasts. Biochem Biophys Res Commun 2003;305:211-4

Liu XD, Zhu YK, Umino T, Spurzem JR, Romberger DJ, Wang $\mathrm{H}$, Reed E, Rennard SI. Cigarette smoke inhibits osteogenic differentiation and proliferation of human osteoprogenitor cells in monolayer and three-dimensional collagen gel culture. J Lab Clin Med 2001;137:208-19

Matsuo K, Zhao C, Peng L, Laplace C, Wang KZQ, Bachler MA, 
Amano $\mathrm{H}$, Aburatani $\mathrm{H}$, Ishikawa $\mathrm{H}$, Wagner EF. Nuclear factor of activated T-cells (NFAT) rescues osteoclastogenesis in precursors lacking c-Fos. J Biol Chem 2004;279:26475-80

Merkel KD, Erdmann JM, McHugh KP, Abu-Amer Y, Ross FP, Teitelbaum SL. Tumor necrosis factor- $\alpha$ mediates orthopedic implant osteolysis. Am J Pathol 1999;154: 203-10

Miyamoto T, Arai F, Ohneda O, Takagi K, Anderson DM, Suda $T$. An adherent condition is required for formation of multinuclear osteoclasts in the presence of macrophage colony-stimulating factor and receptor activator of nuclear factor kappa B ligand. Blood 2000;96:4335-43

Miyaura C, Inada M, Matsumoto C, Ohshiba T, Uozumi N, Shimzu T, Ito A. An essential role of cytosolic phospholipase $\mathrm{A} 2 \alpha$ in prostaglandin E2-mediated bone resorption associated with inflammation. J Exp Med 2003;197:1303-10

Park H, Park OJ, Shin J, Choi Y. Receptor activator of NF- $\kappa B$ ligand enhances the activity of macrophages as antigen presenting cells. Exp Mol Med 2005;37:524-32

Redlich K, Hayer S, Ricci R, David JP, Tohidast-Akrad M, Kollias G, Steiner G, Smolen JS, Wagner EF, Schett G. Osteoclasts are essential for TNF- $\alpha$-mediated joint destruction. J Clin Invest 2002;110:1419-27

Suda T, Takahashi N, Udagawa N, Jimi E, Gillespie MT, Martin TJ. Modulation of osteoclast differentiation and function and ligand families. Endocr Rev 1999;20:345-57

Takahashi K, Ouyang X, Komatsu K, Nakamura N, Hattori M, Baba A, Azuma J. Sodium tanshinone IIA sulfonate derived from Danshen (Salvia miltiorrhiza) attenuates hypertrophy induced by angiotensin II in cultured neonatal rat cardiac cells. Biochem Pharmacol 2002;64:745-9

Takayanagi H, Kim S, Koga T, Nishina H, Isshiki M, Yoshida H, Saiura A, Isobe M, Yokochi T, Inoue J, Wagner EF, Mak TW, Kodama T, Taniguchi T. Induction and activation of the transcription factor NFATc1 (NFAT2) integrate RANKL signaling in terminal differentiation of osteoclasts. Dev Cell 2002;3:889901

Takayanagi $\mathrm{H}$. Mechanistic insight into osteoclast differentiation in osteoimmunology. J Mol Med 2005;83:170-9

Teitelbaum SL, Ross FP. Genetic regulation of osteoclast development and function. Nat Rev Genet 2003;4 :638-49

Walsh MC, Choi Y. Biology of the TRANCE axis. Cytokine Growth Factor Rev 2003;14:251-63

Wang X, Wei Y, Yuan S, Liu G, Lu Y, Zhang J, Wang W. Potential anticancer activity of tanshinone IIA against human breast cancer. Int J Cancer 2005;116:799-807

Whitmarsh AJ, Shore P, Sharrocks AD, Davis RJ. Integration of MAP kinase signal transduction pathways at the serum response element. Science 1995;269:403-7

Wu YJ, Hong CY, Lin SJ, Wu P, Shiao MS. Increase of vitamin $E$ content in LDL and reduction of atherosclerosis in cholesterol-fed rabbits by a water-soluble antioxidant- rich fraction of Salvia miltiorrhiza. Arterioscler Thromb Vasc Biol 1998; 18:481-6 